\title{
GMR
}

\section{Interleukin-4 regulates macrophage polarization via the MAPK signaling pathway to protect against atherosclerosis}

\author{
X.N. Zhao', Y.N. Li ${ }^{2}$ and Y.T. Wang' \\ 1'Department of Geriatric Cardiology, Chinese PLA General Hospital, Beijing, China \\ 2Department of Geriatric Gastroenterology, Chinese PLA General Hospital, Beijing, \\ China \\ Corresponding author: Y.T. Wang \\ E-mail: wangyutang5337@126.com \\ Genet. Mol. Res. 15 (1): gmr.15017348 \\ Received July 30, 2015 \\ Accepted November 4, 2015 \\ Published February 19, 2016 \\ DOI http://dx.doi.org/10.4238/gmr.15017348
}

ABSTRACT. Our study aimed to investigate the effects of interleukin-4 (IL4) on macrophage polarization, as well as its role in the development of atherosclerosis. Human peripheral blood mononuclear cells (PBMCs) were isolated and randomly divided into 3 groups: control group, ox-LDL group, and ox-LDL + IL-4 groups. The expression of M1/M2 macrophage surface markers such as TNF- $\alpha$, CD68, and CD206 were analyzed by western blot. Cell viability was determined using the MTT assay. Measurement of CD86/ CD206 expression ratio (M1/M2 ratio) was performed via flow cytometry. In addition, $\mathrm{ApoE}^{--}$mice on a C57BL/6 background were subjected to high-fat diets, and were used as a model of atherosclerosis. Atherosclerotic lesion area was quantified after mice were treated with ox-LDL and IL-4. Finally, expression of phosphorylated MAPK signaling molecules such as $p$-ERK and $p-J N K$ was quantified using western blot. The expression of TNF- $\alpha$ and CD86 markedly increased after cells were treated with ox-LDL, whereas the expression of CD206 markedly increased after PBMCs were treated with IL-4. It is possible that IL-4 could decrease ox-LDL-induced cell viability and the CD86/CD206 (M1/M2) ratio. Additionally, IL-4 intervention attenuated 
ox-LDL-induced atherosclerotic lesions in $\mathrm{ApoE}^{-/-}$mice, and decreased oxLDL-induced expression of $\mathrm{p}-\mathrm{ERK}$ and $\mathrm{p}$-JNK. Our findings indicate that IL-4 may induce macrophages to take on an M2 phenotype in order to resolve inflammation via inhibition of MAPK signaling pathways, thereby protecting against atherosclerosis. IL-4 may serve as an intervention target for atherosclerosis.

Key words: Atherosclerosis; Interleukin-4; M2 macrophage; MAPK signaling pathway

\section{INTRODUCTION}

Atherosclerosis is a chronic inflammatory disease due to abnormal accumulation of macrophages, white blood cells, and lipids in the arterial walls (Hansson et al., 2006). It is characterized by endothelial dysfunction, vascular inflammation, plaque formation, and diminished oxygen supply to target organs (Davignon and Ganz, 2004; Mannarino and Pirro, 2008). Atherosclerosis can lead to various cardiovascular diseases, which are considered to be major contributors of death worldwide (Cooper et al., 2000; Glass and Witztum, 2001). To this date, the molecular mechanisms underlying the pathogenesis of atherosclerosis remain largely unknown.

Accumulation of macrophages in the vascular wall is a hallmark of atherosclerosis, and plays a key role in the development of atherosclerotic lesions (Adamson and Leitinger, 2011; Moore and Tabas, 2011). Macrophages can alter their phenotype in response to plaque lipids, and this phenotypic modulation has been shown to influence both progression and regression of atherosclerosis (Adamson and Leitinger, 2011). Moreover, increasing evidence suggests that inflammation also plays an important role in all phases of atherosclerosis (Siegel et al., 2013). Macrophages in advanced atherosclerotic plaques have been shown to be major contributors of the inflammatory response via secretion of pro-inflammatory mediators (Moore et al., 2013). Distinguishable macrophage phenotypes, $M 1$ and $M 2$, were observed during the course of inflammatory reactions (Martinez et al., 2009). Therefore, a better understanding of the regulatory mechanism of macrophage polarization will be important for the development of potential therapeutic strategies.

Interleukin-4 (IL-4) is a well-known anti-inflammatory cytokine. IL-4 has been shown to play an important role in the progression of atherosclerosis (Davenport and Tipping, 2003). IL-4 deficiency can decrease atherosclerotic lesion formation in a site-specific manner in female LDL receptor ${ }^{--}$mice (King et al., 2002). In addition, IL-4 with anti-inflammatory functions can steer macrophages towards an M2 phenotype during inflammations (Martinez et al., 2009). M2 macrophages can reduce pro-inflammatory cytokine secretion and produce anti-inflammatory factors to dampen inflammatory responses (Peiser and Gordon, 2001). The phenotype switch of M2 macrophages from an anti- to a pro-inflammatory state can have important consequences for development of atherosclerosis (van Tits et al., 2011). Despite these observations, it is unknown whether IL-4 can inhibit atherosclerosis via regulation of macrophage polarization.

The uptake of oxidized low-density lipoprotein (ox-LDL) by macrophages is believed to play a pivotal role in the development of atherosclerosis (Dai et al., 2014). In the current study, we isolated human peripheral blood mononuclear cells (PBMCs) to investigate the effects of IL-4 on macrophage polarization. In addition, we subjected apolipoprotein $E(A p o E)^{-/-}$mice to high-fat 
diets, thus making them models of atherosclerosis. The atherosclerotic lesion area was quantified after treatments with ox-LDL and recombinant IL-4. Our study aims to investigate the effects of IL-4 on macrophage polarization, as well as to elucidate its roles in atherosclerosis development. Our study provides novel insights into the roles of IL-4 as a potential therapeutic for atherosclerosis.

\section{MATERIAL AND METHODS}

\section{Cell isolation, culture and treatment}

PBMCs were isolated from blood by density centrifugation using Ficoll-Paque ${ }^{\mathrm{TM}}$ PLUS (GE, Piscataway, NJ, USA). Cells were then washed and resuspended in RPMI 1640 medium containing $10 \%$ fetal calf serum (FCS). Cells (5 million cells $/ \mathrm{mL}$ ) were seeded on 96 -well flatbottom microtiter plates, and were divided into 3 groups: control group, ox-LDL group, and ox-LDL + IL-4 groups. Cells were cultured in RPMI-10\% FCS medium for 7 days.

\section{Western blot}

All cells were lysed on ice with radio immunoprecipitation assay (RIPA) lysis buffer containing protease inhibitors (BestBio, Shanghai, China). An equal amount of proteins were subjected to electrophoresis on a sodium dodecyl sulfate-polyacrylamide gel, and then transferred to a nitrocellulose membrane. After the membrane was blocked for non-specific binding, it was probed with the primary antibodies for TNF- $\alpha$, CD68 and CD206 (Cell Signaling), followed by secondary antibodies conjugated to horseradish peroxidase. Membranes were probed with $\beta$-actin antibody as an internal control. Finally, proteins were detected using an enhanced chemiluminescence kit (Millipore). All experiments were performed three times for reproducibility. Images were obtained using infrared scanning (Odyssey, USA), and quantified using the GeIPro32 program. Protein expression was normalized to $\beta$-actin.

\section{MTT assay}

Cells were seeded on 96-well plates at a density of $2 \times 10^{4}$ cells/well, and cell viability was determined using the MTT assay (Sigma Chemicals, Perth, WA, Australia). In brief, MTT (10 $\mathrm{mg} / \mathrm{mL}$ ) was added into each sample-containing well, and incubated for $4 \mathrm{~h}$. Isopropanol (in 0.04 $\mathrm{N}-\mathrm{HCl}$ ) was added to dissolve the formazan crystals. Each experiment was performed in triplicates. Absorbance was read at $570 \mathrm{~nm}$ using an automatic microplate reader (BioTek Instruments, Inc.). Relative cell viability was measured as the relative absorbance of each sample normalized to control, and is reported as percentage.

\section{Flow cytometry}

Measurements of cell surface marker expression (mean fluorescence intensity) were performed by flow cytometry (FACS Calibur, BD Biosciences, San Jose, CA, USA). In brief, cells were incubated with specific antibodies for CD86 and CD206 (BD Biosciences Pharmingen ${ }^{\mathrm{TM}}$, San Diego, CA, USA). Samples were analyzed on an automated immunoanalyzer (Immulite 1000 Immunoassay System, Siemens, Schweiz). The M1/M2 ratio, defined as the CD86/CD206 expression ratio, was calculated. 


\section{Animals and diets}

Atherosclerosis was shown to be induced in $\mathrm{ApoE}^{-/-}$mice under high-fat diets (Madhur et al., 2011). In our study, ApoE ${ }^{-/-}$mice with a C57BL/6 genetic background were bred in pathogenfree environment with free access to food and water. At the age of 10 weeks, 16 male mice were randomly divided into two groups ( $N=8$ per group): the control group was fed high-fat diet containing ox-LDL for 12 weeks, while the experimental group was fed high-fat diet plus both oxLDL and recombinant IL-4 at the same time. All procedures were performed following the National Institutes of Health Guidelines for the Use of Laboratory Animals, and were approved by the local council of Ethics Committee.

\section{Quantification of atherosclerotic lesion area}

After removing the adventitial fatty tissue, aortas from the aortic root to the renal artery were longitudinally opened, and fixed in 10\% formalin for $36 \mathrm{~h}$. After staining with Sudan IV for 10 min, the fixed aortas were differentiated in $70 \%$ alcohol for $15 \mathrm{~min}$, and washed in water for $30 \mathrm{~min}$. Finally, the stained aortas were imaged with a digital camera connected to a dissection microscope. Area of the atherosclerotic lesion in the entire aorta and the aortic arch was evaluated as the ratio of the positively stained area to the total aortic area using the Image-Pro Plus 6.0 software.

\section{Phosphorylation of MAPK signaling molecules via western blot analysis}

In order to explore regulatory mechanism of IL-4 in the development of atherosclerosis, phosphorylation of MAPK signaling molecules including ERK (p42) and JNK (p46) was quantified via western blot analysis. Aortic tissues were ground on ice in RIPA buffer, and aortic proteins were isolated by centrifugation at $12,000 \mathrm{~g}$ for $20 \mathrm{~min}$. Primary antibodies for $\mathrm{p}$-ERK and $\mathrm{p}$-JNK were obtained from Cell Signaling Technology. Western blot was performed as described above.

\section{Statistics analysis}

All collected data are reported as means $\pm S D$, and were tested for normal distribution using the one-sample K-S test. Measured data were analyzed using the Student $t$-test (for two groups) or one-way ANOVA (for more than three groups). Between-group comparisons were performed using the post hoc Tukey test in ANOVA. Statistical significance was defined when $\mathrm{P}<0.05$.

\section{RESULTS}

\section{Surface marker expression of M1/M2 macrophage}

Western blots showed that expression of TNF- $\alpha$ and CD86 was markedly increased after PBMCs were treated with ox-LDL only $(P<0.05)$. However, TNF- $\alpha$ and CD86 expression in ox-LDL and IL-4 treated PBMCs did not differ from that of control $(P>0.05$; Figure $1 A$ and $B)$, suggesting that ox-LDL update could induce polarization of monocytes towards the M1 phenotype. On the other hand, as shown in Figure 1C, expression of CD206 markedly increased after PBMCs were treated with both ox-LDL and IL-4 $(\mathrm{P}<0.05)$. When treated with only ox-LDL, CD206 expression 
was similar $(P>0.05)$ to that of control (Figure 1A and B), suggesting that IL-4 could induce polarization of monocytes towards an M2 phenotype.
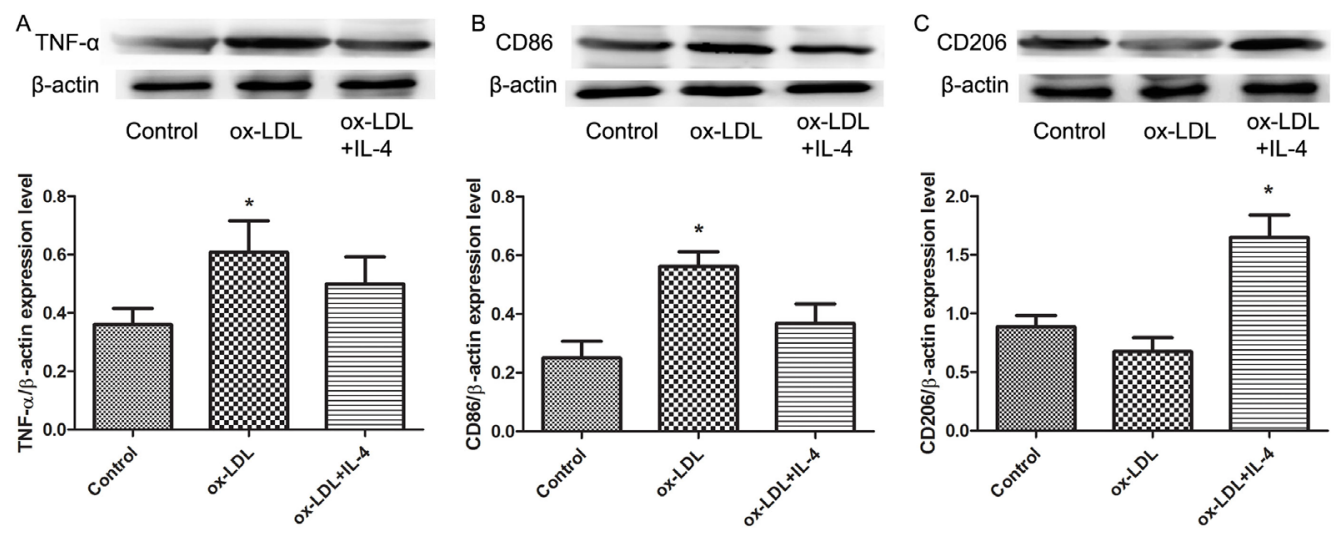

Figure 1. Expression of surface markers of M1/M2 macrophages following treatment with ox-LDL and IL-4. A. Expression of TNF- $\alpha$. B. Expression of CD86. C. Expression of CD206. Results are reported as means \pm $\mathrm{SD} ;{ }^{*} \mathrm{P}<0.05$ compared to the control group.

\section{IL-4 may inhibit the elevated cell viability induced by ox-LDL}

Figure 2 shows cell viability $72 \mathrm{~h}$ following the addition of drugs. Results indicated that uptake of ox-LDL could significantly promote cell viability as compared with the control group. On the other hand, cell viability of the ox-LDL + IL-4 group was lower than that of the ox-LDL group, suggesting that IL-4 could inhibit the elevated cell viability induced by ox-LDL.

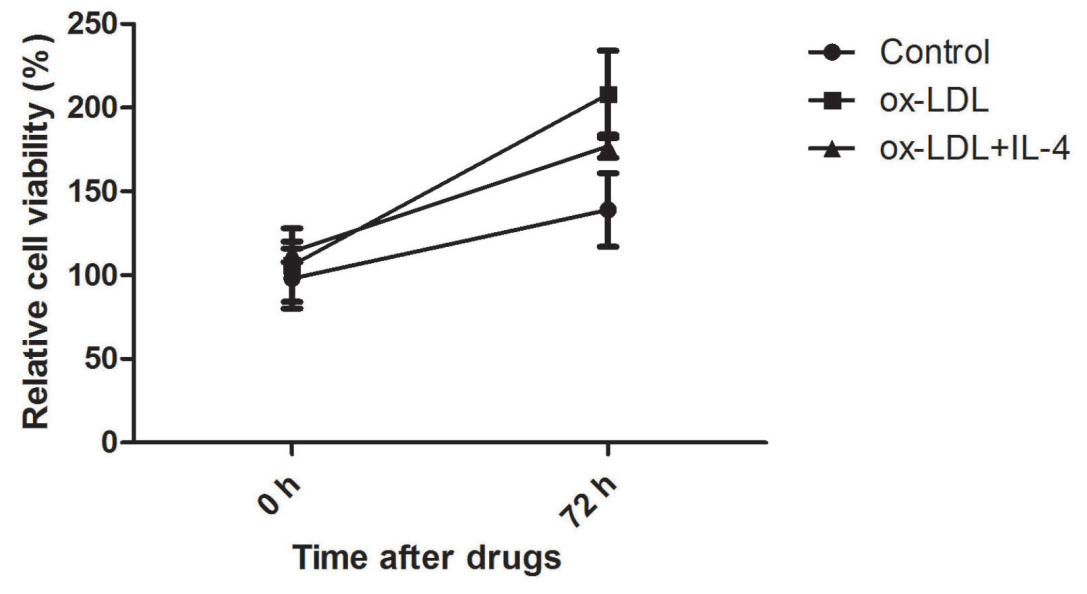

Figure 2. Cell viability after $72 \mathrm{~h}$ following ox-LDL and IL-4 treatment. 


\section{Regulation of macrophage phenotypic polarization by ox-LDL and IL-4}

To further verify the regulation of ox-LDL and IL-4 on macrophage phenotypic polarization, flow cytometry was performed to detect the CD86/CD206 (M1/M2) ratio (Figure 3). Results indicated that uptake of ox-LDL significantly increased the M1/M2 ratio $(P<0.05)$ as compared with control, and this ratio decreased following treatment of both ox-LDL and IL-4. This further suggests that ox-LDL could induce polarization of monocytes towards an M1 phenotype, and the addition of IL-4 induced them towards an M2 phenotype.

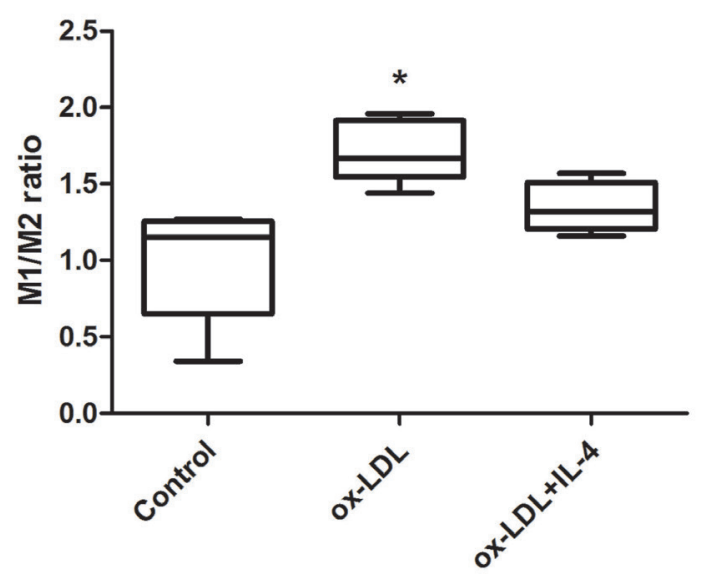

Figure 3. CD86/CD206 (M1/M2) ratio. Results are reported as means $\pm \mathrm{SD}$; ${ }^{*} \mathrm{P}<0.05$ compared to the control group.

\section{IL-4 reduced atherosclerotic lesion formation in $A p o E^{-/-}$mice}

Sudan IV staining showed the percentage of atherosclerotic plaques in the entire aorta as well as the aortic arch (Figure 4A and B). IL-4 significantly reduced atherosclerotic lesion in both the entire aorta and the aortic arch of $\mathrm{ApoE}^{-/-}$mice as compared with those treated with ox-LDL alone $(P<0.05)$.
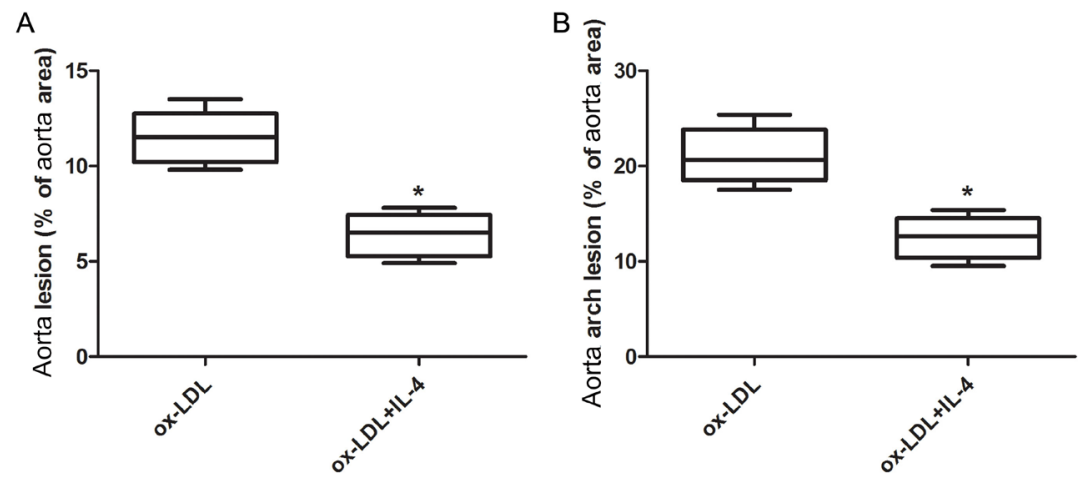

Figure 4. Sudan IV staining. Percentage of atherosclerotic lesion in the (A) entire aorta and (B) the aorta arch. Results are reported as means $\pm S D$ and ${ }^{*} \mathrm{P}<0.05$ compared to the control group. 


\section{Effect of IL-4 on the phosphorylation of MAPK signaling pathway}

Phosphorylation of MAPK signaling molecules was measured to determine the effect of IL-4 on atherosclerosis development (Figure 5A and B). The results showed that expression levels of p-ERK and p-JNK in the ox-LDL group were significantly increased as compared with the control group. However, their expression levels in the ox-LDL + IL-4 group were significantly decreased as compared with the ox-LDL group, but increased as compared with the control group. These results indicate that IL-4 may play a pivotal role in regulating inflammation via inhibition of MAPK signaling pathways.
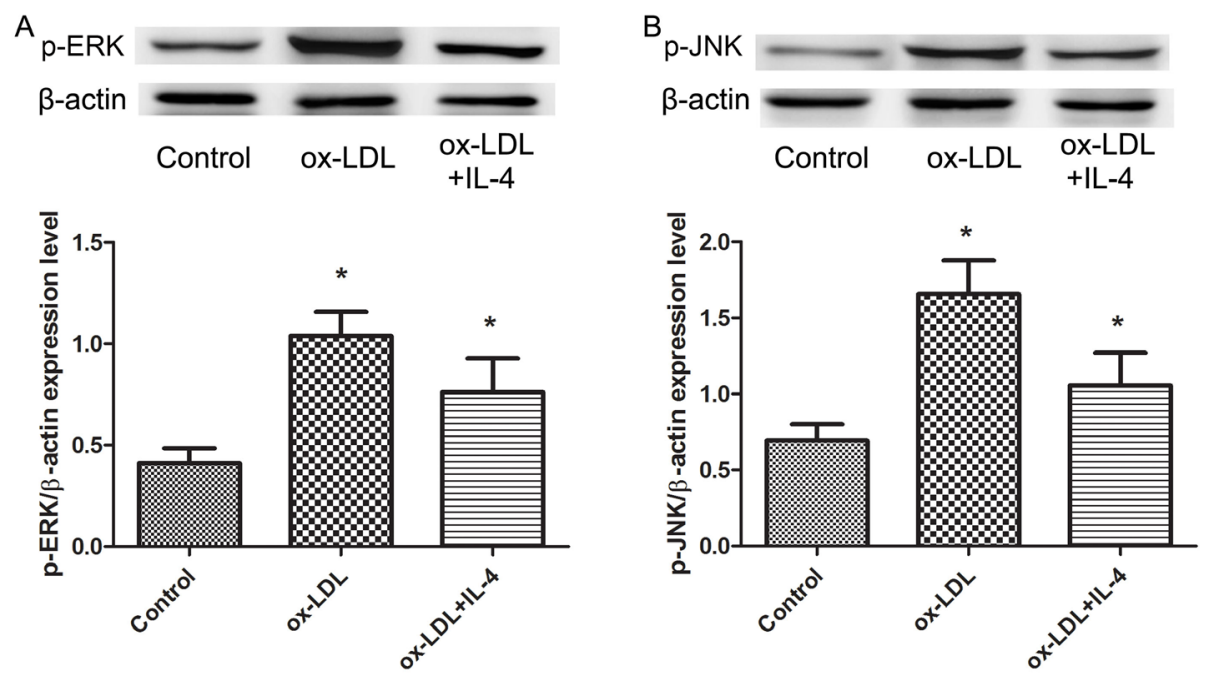

Figure 5. Phosphorylation of MAPK proteins. Expression levels of phosphorylated (A) ERK and (B) JNK. Results are reported as means $\pm S D$ and ${ }^{*} P<0.05$ compared to the control group.

\section{DISCUSSION}

A better understanding of the key mechanism involved in the pathogenesis of atherosclerosis will be important for implementing potential therapeutic strategies. We found that IL-4 could induce polarization of monocytes towards an M2 phenotype. Moreover, IL-4 intervention attenuated the ox-LDL-induced atherosclerotic lesion in $\mathrm{ApoE}^{--}$mice, and decreased the ox-LDLinduced expression level of p-ERK and p-JNK. These findings suggest that IL-4 might play a pivotal role in protection against atherosclerosis via inhibition of the MAPK signaling pathway.

Macrophages that accumulate in atherosclerotic plaques lead to failure to resolve inflammation, and thus contribute to the progression of these lesions to more advanced plaques (Randolph, 2008). Previous study has shown that the M1 macrophages can promote plaque inflammation, while M2 macrophages can resolve plaque inflammation in models of atherosclerosis (Moore et al., 2013). Activation of M2 macrophage was originally discovered as a response to IL-4 (Gordon, 2003). M2 macrophage is stimulated by IL-4 and up-regulates expression of the mannose receptor CD206 (Moore et al., 2013). In our study, IL-4 intervention markedly increased expression of CD206 and decreased the CD86/CD206 ratio in PBMCs. Our results are in line with previous findings, which suggest that IL-4 induced macrophages to progress towards an M2 phenotype. 
M2 macrophage could secrete several anti-inflammatory cytokines including IL-10 (Mantovani et al., 2002). IL-10, a prototypical anti-inflammatory cytokine, was reported as an "Immunologic Scalpel" for atherosclerosis (Terkeltaub, 1999). In addition, M2 macrophage could express several transcription factors such as Krüppel-like factor 4 (KLF4) and peroxisome proliferator activated receptor- $\gamma$ (PPAR $\gamma$ ) (Moore et al., 2013). KLF4 was shown to be associated with the formation and development of atherosclerosis, and may serve as a novel target for the prevention and cure of this disease (Yan F-f et al., 2008) (Yan et al., 2008). PPAR $\gamma$ was also confirmed to activate thiazolidinediones, and thus offer protection against atherosclerosis through inhibition of vascular cell growth and movement (Hsueh and Law, 2001). In our study, IL-4 intervention inhibited oxLDL-induced cell viability and attenuated ox-LDL-induced atherosclerotic lesion in ApoE-/- mice. Therefore, we speculate that IL-4 may induce macrophages towards an M2 phenotype to resolve inflammation, thereby playing a pivotal role in protection against atherosclerosis.

To further explore the regulatory mechanism of IL-4 in the pathogenesis of atherosclerosis, phosphorylation of MAPK signaling molecules was determined. In previous findings, p38 MAPK has been shown to be a potentially important mediator in promoting atherosclerosis (Seimon et al., 2009), and p38 pathway kinases may function as anti-inflammatory drug targets for inflammatory diseases (Schindler et al., 2007). In addition, leukocyte cell-derived chemotaxin 2 (LECT2) can also play a role in atherosclerotic inflammatory reactions via JNK phosphorylation (Hwang et al., 2015). In addition, the DPP-4 inhibitor sitagliptin can reduce leukocyte-endothelial cell interactions and inflammation, and attenuate atherosclerosis progression via AMPK- and MAPK-dependent mechanisms (Zeng et al., 2014). Haeng Park et al. (2014) also confirmed that the anti-atherosclerotic effects of Polygonum aviculare L. ethanol extract in $\mathrm{ApoE}^{-/-}$mice are mediated via the MAPK pathway. Furthermore, high levels of IL-4 in the tumor microenvironment were observed, which could activate MAPKs, including ERK, p38, and JNK (Roca et al., 2012). Jiménez-Garcia et al. (2015) demonstrated that p38 MAPK could play a pivotal role in IL-4-induced alternative activation of macrophages. Our results showed that IL-4 intervention markedly decreased ox-LDL-induced expression of $p-E R K$ and p-JNK, suggesting that IL-4 may induce activation of M2 macrophages to protecting against atherosclerosis via inhibition of MAPK signaling pathways.

In conclusion, IL-4 may induce macrophages towards an M2 phenotype to resolve inflammation via inhibition of MAPK signaling pathways. IL-4 may serve as an intervention target for atherosclerosis. Moreover, it is possible that early intervention using a combination of antiinflammatory cytokines and the inhibitors of pro-inflammatory cytokines may achieve good outcomes for atherosclerosis in a clinical setting. However, further studies are needed to verify our findings.

\section{Conflicts of interest}

The authors declare no conflict of interest.

\section{REFERENCES}

Adamson S and Leitinger N (2011). Phenotypic modulation of macrophages in response to plaque lipids. Curr. Opin. Lipidol. 22: 335-342. http://dx.doi.org/10.1097/MOL.0b013e32834a97e4

Cooper R, Cutler J, Desvigne-Nickens P, Fortmann SP, et al. (2000). Trends and disparities in coronary heart disease, stroke, and other cardiovascular diseases in the United States: findings of the national conference on cardiovascular disease prevention. Circulation 102: 3137-3147. http://dx.doi.org/10.1161/01.CIR.102.25.3137

Dai Y, Dai D, Wang X, Ding Z, et al. (2014). GLP-1 agonists inhibit ox-LDL uptake in macrophages by activating protein kinase A. J. Cardiovasc. Pharmacol. 64: 47-52. http://dx.doi.org/10.1097/FJC.0000000000000087 
Davenport P and Tipping PG (2003). The role of interleukin-4 and interleukin-12 in the progression of atherosclerosis in apolipoprotein E-deficient mice. Am. J. Pathol. 163: 1117-1125. http://dx.doi.org/10.1016/S0002-9440(10)63471-2

Davignon J and Ganz P (2004). Role of endothelial dysfunction in atherosclerosis. Circulation 109 (Suppl 1): III27-III32. http:// dx.doi.org/10.1161/01.CIR.0000131515.03336.f8

Glass CK and Witztum JL (2001). Atherosclerosis. the road ahead. Cell 104: 503-516. http://dx.doi.org/10.1016/S00928674(01)00238-0

Gordon S (2003). Alternative activation of macrophages. Nat. Rev. Immunol. 3: 23-35. http://dx.doi.org/10.1038/nri978

Haeng Park SH, Sung Y-Y, Nho KJ and Kim HK (2014). Anti-atherosclerotic effects of Polygonum aviculare L. ethanol extract in ApoE knock-out mice fed a Western diet mediated via the MAPK pathway. J. Ethnopharmacol. 151: 1109-1115. http:// dx.doi.org/10.1016/j.jep.2013.12.021

Hansson GK, Robertson A-KL and Söderberg-Nauclér C (2006). Inflammation and atherosclerosis. Annu. Rev. Pathol. 1: 297329. http://dx.doi.org/10.1146/annurev.pathol.1.110304.100100

Hsueh WA and Law RE (2001). PPARgamma and atherosclerosis: effects on cell growth and movement. Arterioscler. Thromb. Vasc. Biol. 21: 1891-1895. http://dx.doi.org/10.1161/hq1201.100261

Hwang H-J, Jung TW, Hong HC, Seo JA, et al. (2015). LECT2 induces atherosclerotic inflammatory reaction via CD209 receptor-mediated JNK phosphorylation in human endothelial cells. Metabolism 64: 1175-1182. http://dx.doi.org/10.1016/j. metabol.2015.06.001

Jiménez-Garcia L, Herránz S, Luque A and Hortelano S (2015). Critical role of p38 MAPK in IL-4-induced alternative activation of peritoneal macrophages. Eur. J. Immunol. 45: 273-286. http://dx.doi.org/10.1002/eji.201444806

King VL, Szilvassy SJ and Daugherty A (2002). Interleukin-4 deficiency decreases atherosclerotic lesion formation in a site-specific manner in female LDL receptor-/- mice. Arterioscler. Thromb. Vasc. Biol. 22: 456-461. http://dx.doi.org/10.1161/hq0302.104905

Madhur MS, Funt SA, Li L, Vinh A, et al. (2011). Role of interleukin 17 in inflammation, atherosclerosis, and vascular function in apolipoprotein e-deficient mice. Arterioscler. Thromb. Vasc. Biol. 31: 1565-1572. http://dx.doi.org/10.1161/ ATVBAHA.111.227629

Mannarino E and Pirro M (2008). Molecular biology of atherosclerosis. Clin. Cases Miner. Bone Metab. 5: 57-62.

Mantovani A, Sozzani S, Locati M, Allavena P, et al. (2002). Macrophage polarization: tumor-associated macrophages as a paradigm for polarized M2 mononuclear phagocytes. Trends Immunol. 23: 549-555. http://dx.doi.org/10.1016/S1471$\underline{4906(02) 02302-5}$

Martinez FO, Helming L and Gordon S (2009). Alternative activation of macrophages: an immunologic functional perspective. Annu. Rev. Immunol. 27: 451-483. http://dx.doi.org/10.1146/annurev.immunol.021908.132532

Moore KJ and Tabas I (2011). Macrophages in the pathogenesis of atherosclerosis. Cell 145: 341-355. http://dx.doi. org/10.1016/j.cell.2011.04.005

Moore KJ, Sheedy FJ and Fisher EA (2013). Macrophages in atherosclerosis: a dynamic balance. Nat. Rev. Immunol. 13: 709-721. http://dx.doi.org/10.1038/nri3520

Peiser L and Gordon S (2001). The function of scavenger receptors expressed by macrophages and their role in the regulation of inflammation. Microbes Infect. 3: 149-159. http://dx.doi.org/10.1016/S1286-4579(00)01362-9

Randolph GJ (2008). Emigration of monocyte-derived cells to lymph nodes during resolution of inflammation and its failure in atherosclerosis. Curr. Opin. Lipidol. 19: 462-468. http://dx.doi.org/10.1097/MOL.0b013e32830d5f09

Roca H, Craig MJ, Ying C, Varsos ZS, et al. (2012). IL-4 induces proliferation in prostate cancer PC3 cells under nutrientdepletion stress through the activation of the JNK-pathway and survivin up-regulation. J. Cell. Biochem. 113: 1569-1580.

Schindler JF, Monahan JB and Smith WG (2007). p38 pathway kinases as anti-inflammatory drug targets. J. Dent. Res. 86: 800-811. http://dx.doi.org/10.1177/154405910708600902

Seimon TA, Wang Y, Han S, Senokuchi T, et al. (2009). Macrophage deficiency of p38 $\alpha$ MAPK promotes apoptosis and plaque necrosis in advanced atherosclerotic lesions in mice. J. Clin. Invest. 119: 886-898.

Siegel D, Devaraj S, Mitra A, Raychaudhuri SP, et al. (2013). Inflammation, atherosclerosis, and psoriasis. Clin. Rev. Allergy Immunol. 44: 194-204. http://dx.doi.org/10.1007/s12016-012-8308-0

Terkeltaub RA (1999). IL-10: An "immunologic scalpel" for atherosclerosis? Arterioscler. Thromb. Vasc. Biol. 19: $2823-2825$. http://dx.doi.org/10.1161/01.ATV.19.12.2823

van Tits LJ, Stienstra R, van Lent PL, Netea MG, et al. (2011). Oxidized LDL enhances pro-inflammatory responses of alternatively activated M2 macrophages: a crucial role for Krüppel-like factor 2. Atherosclerosis 214: 345-349. http:// dx.doi.org/10.1016/j.atherosclerosis.2010.11.018

Yan FF, Liu YF, Liu Y and Zhao YX (2008). KLF4: a novel target for the treatment of atherosclerosis. Med. Hypotheses 70: 845-847. http://dx.doi.org/10.1016/j.mehy.2007.07.031

Zeng Y, Li C, Guan M, Zheng Z, et al. (2014). The DPP-4 inhibitor sitagliptin attenuates the progress of atherosclerosis in apolipoprotein-E-knockout mice via AMPK- and MAPK-dependent mechanisms. Cardiovasc. Diabetol. 13: 32. http:// dx.doi.org/10.1186/1475-2840-13-32 